\title{
La teoría de la complejidad y los desafíos docentes en las IEMS y Superior en Quintana Roo
}

\author{
Daniela Díaz Pérez \\ Correo electrónico: diazperezdaniela0911@gmail.com \\ ORCID: https://orcid.org/0000-0003-1975-7727 \\ Escuela Superior de Comercio y Administración, Unidad \\ Tepepan, IPN \\ María Nathaly Xiu Sánchez \\ Correo electrónico: nathalyxiu03@gmail.com \\ ORCID: https://orcid.org/0000-0002-0704-8125 \\ Instituto Tecnológico Superior de Felipe Carrillo Puerto, \\ TecNM
}

\author{
Angélica Reyes Mendoza \\ Sociales, Tecate, B.C. \\ Recepción: 03/09/2021 \\ Aceptación: 19/10/2021
}

Correo electrónico: angelita@uabc.edu.mx

ORCID: http://orcid.org/0000-0002-4926-861X

Facultad de Ciencias de la ingeniería Administrativas y

\section{Resumen}

La educación tiene un papel importante en la vida de las personas y en la sociedad en general, por lo cual se requiere analizar cómo la pandemia afectó a las Instituciones de Educación Media Superior y/o Superior en todo el mundo, a la luz de la teoría de la complejidad. La presente investigación se llevó a cabo en Felipe Carrillo Puerto, Quintana Roo, con una muestra representativa de 66 docentes, la metodología fue de tipo cuantitativa, con un diseño transaccional y un alcance correlacional, la variable independiente fue los desafíos en sus dimensiones (entorno innovador, retos inesperados, liderazgo) y la variable dependiente gestión de proyectos con sus dimensiones (producto, proceso, personal, sistemas), con la finalidad de impactar en la educación a través de la teoría de la complejidad denotando en primera instancia los principales desafíos a los que se enfrentan los docentes los cuales fueron los retos inesperados en un $75 \%$ que comprenden la forma de impartir clases y la manera de relacionarse con los alumnos, en segundo lugar con un $69 \%$ el liderazgo relacionado con la motivación a los alumnos y cómo a través de la adecuada gestión se puede mejorar en esta época de contingencia.

Palabras Clave: A2 Educación, I9 contingencia, B3 teoría de la complejidad y L1 Gestión de proyectos.

\section{Abstract}

Education plays an important role in people's lives and in society in general, which is why it is necessary to analyze how the pandemic affected Institutions of Higher and 
/ or Higher Education throughout the world, in light of the complexity theory. This research was carried out in Felipe Carrillo Puerto, Quintana Roo, with a representative sample of 66 teachers, the methodology was quantitative, with a transactional design and a correlational scope, the independent variable was the challenges in its dimensions (environment innovative, unexpected challenges, leadership) and the dependent variable project management with its dimensions (product, process, personnel, systems), in order to impact education through complexity theory denoting in the first instance the main challenges to which teachers face which were the unexpected challenges in $75 \%$ that understand the way of teaching and the way of relating to students, in second place with $69 \%$ leadership related to the motivation of students and how through proper management it can be improved in this time of contingency.

Key Words: A2 Education, I9 contingency, B3 complexity theory and L1 Project management.

\section{Introducción}

Ante los momentos que se viven hoy por hoy en la llegada de la pandemia, es necesario que se empleen estrategias en las Instituciones de Educación Media y/o Superior, para que tanto los docentes como los estudiantes desarrollen habilidades que les permitan gestionar sus clases de manera innovadora en donde demuestren un buen desempeño ante los retos para enfrentar esta situación de la manera más óptima en sus lugares de residencia. Para ello se pretende realizar una investigación que coadyuve a conocer los principales desafíos (entorno innovador, retos inesperados, liderazgo) y cómo ellos, afectan a la gestión de proyectos (producto, proceso, personal, sistemas) de tal manera que brinde estos conocimientos a las instituciones y den las bases para contribuir en la mejora de la gestión de los mismos en medio de esta situación y en momentos futuros. La teoría en este caso la de la complejidad es de utilidad para que los profesionales realicen con eficacia y eficiencia la acción de administrar, donde a través de observar la complejidad del fenómeno organizacional en tiempos de la sociedad del conocimiento en red y de la crisis ambiental se logre afrontar dicha situación (Arias-Pineda y Ramirez-Martinez, 2019).

El objetivo de esta investigación impactar en la educación a través de la teoría de la complejidad denotando en primera instancia los principales desafíos a los que se enfrentan los docentes de las Instituciones de Educación Media y/o Superior derivados de la contingencia mediante la gestión de proyectos en el ámbito educativo con la llegada de la pandemia por COVID19.
La gestión de proyectos según PMI (2013), es la aplicación de conocimientos, habilidades, herramientas y técnicas a las actividades del proyecto con el fin de satisfacer sus necesidades. Así mismo Carvalho y Rabechini (2011) complementan esta definición, mencionando la norma ISO 10006, que dice que el manejo del proyecto incluye la planificación, organización, supervisión y control de todos los aspectos del proyecto en un proceso continuo para lograr sus objetivos. Por ejemplo en Estados Unidos de Norteamérica se menciona la aceptación de los principios y prácticas de gestión de proyectos; desarrollar habilidades específicas de planificación y control, incluido el uso de determinados paquetes de software; y mejorar la tasa de éxito de la planificación y ejecución de proyectos (Darchibald, 1989).En este caso se requiere del campo de la gestión de proyectos para apoyar a la educación y a la formación con el objetivo de impactar en la planificación y ejecución de la educación apoyada en las tecnologías de información y comunicación.

Ferrás (2010) afirma que no todos los proyectos son innovación. El autor conceptualiza "proyecto" como una unidad de la innovación, ya que si la organización no tiene una cartera de proyectos de innovación, dice el autor, no es una organización innovadora, independientemente de su estrategia, sus capacidades y de sus proyectos. Afirma que un

Proyecto de innovación debe cumplir con dos requisitos: debe ser capaz de generar ventajas competitivas y debe incorporar el riesgo (principalmente tecnológico o financiero) en la naturaleza.

Para efectos del abordaje de la teoría de la complejidad, Barberousse (2008) opina, que la propuesta del pensamiento complejo ha cuestionado no sólo el concepto mismo de ciencia, sino que ha proclamado que lo que ha variado es la naturaleza misma de lo que entendemos por conocimiento. En consecuencia, se verían también afectados los procesos de construcción de saberes y aprendizajes que ocurren en el marco de las mediaciones pedagógicas. En medio del contexto que se enfrenta, es necesario, visualizar otros enfoques y otras respuestas a los desafíos, que en esta instancia, pueden ser apoyados por esta teoría.

En la presente investigación desarrolla su marco teórico con fuentes de información relacionados al tema que se está investigando y que sirven para dar introducción a la misma. Posteriormente se muestra el método llevado a cabo, el cómo planeamos y realizamos la investigación tanto de campo como teórica, luego de esto los resultados obtenidos de toda nuestra muestra, por último las conclusiones generadas luego del análisis estadístico y teórico así como las respectivas fuentes bibliográficas. 


\section{Marco teórico}

La forma actual en la que se desempeña un docente o un alumno, se puede ver con claridad la teoría de la complejidad en donde se presenta novedosos cambios y más aún con la llegada de la contingencia, debido a que se obliga a actuar de manera casi imprevista por las restricciones que ahora pone el ambiente en la educación a distancia y en donde tanto los estudiantes como docentes se ven en la necesidad de encontrar maneras de comunicación e interacción desde casa, esto demanda un trabajo mayor en las Instituciones de Educación Media y/o Superior. Como lo comenta González (2009) que la complejidad, "es una forma de analizar, de reflexionar sobre determinados aspectos de la naturaleza, la sociedad y el pensamiento, los cuales presentan ciertas características que los clasifican como sistemas de comportamiento complejo" Se dice entonces que se ha llegado al caos, teniendo este vocablo una connotación especial en la teoría que estudia la complejidad, en este ámbito, se puede encontrar la relación entre los desafíos que preponderan en la educación en consecuencia de la pandemia y lo que indican las características de la complejidad.

Cervantes (2020) comenta que el cierre de las escuelas reveló un conjunto de desafíos para las autoridades educativas, el profesorado y las familias, en tanto la ejecución de estas medidas se configura en un entramado de circunstancias educativas, sociales y económicas que, en suma, profundizan la desigualdad educativa, lo cual será confirmado con mayor detalle por medio de la investigación para poder actuar ante ello.

Se están realizando cambios en las escuelas que responden a los cambios filosóficos y metodológicos tanto en las ciencias humanas como en las ciencias naturales. La escuela debe atender las necesidades de la comunidad, de sus alumnos y de la sociedad; para ello, por un lado, ha de conocer esta nueva realidad y, por otro, atender a los estudios para y sobre la escuela que se están potenciando fundamentalmente desde las propuestas de la teoría de la complejidad (Cárdenas y Rivera, 2004)

En opinión a estos autores, se da el parteaguas para indicar que la teoría de la complejidad puede ser un medio de búsqueda de una manera de abordar la gestión de proyectos, ante los retos que se presentan y poder enfrentar incluso el devenir en la educación que se encuentra en constante cambio, puesto que como individuos se tiene la capacidad de adaptarse al entorno.

Narváez (2021) indica que la formación si bien ya presentaba algunos retos y obstáculos que se vieron incrementados por la falta de una computadora, internet, porque el estudiantado cambió sus horas clase, por horas-trabajo para ayudar en el gasto familiar y poca presencia en el aula virtual, por lo cual se denota una importante temática a tratar y que se requiere de estrategias que le permitan al docente motivar y ver de alguna manera como apoyar en este sentido. Esto es un poco de los desafíos a los que hoy día enfrentamos en el ámbito educativo con la contingencia COVID-19, sin embargo hay otros aspectos a considerar dentro de la variable independiente (desafíos), no solo a los retos inesperados sino al entorno innovador y al liderazgo que son clave en esta investigación.

"Esta pandemia nos agarra a todos por sorpresa. Los que tenemos más tiempo trabajando con la educación a distancia logramos hacer una movilización rápida, sin embargo, la cantidad de cosas que teníamos que cambiar representó un gran trabajo", aseguró el vicerrector Académico y de Innovación Educativa del Tec de Monterrey.

"Como el tsunami noticioso ocasionado por el virus que se disemina cruzando fronteras, el día 14 de marzo circuló a través de redes sociales, grupos de maestros y canales de comunicación institucional -en ese ordenla conferencia de prensa del secretario de educación pública, Esteban Moctezuma Barragán y el subsecretario de salud, Hugo López Gatell, informando la suspensión de actividades educativas presenciales al 23 del mismo mes. La Secretaría de Educación Pública (SEP) dio a las escuelas siete días para responder un "examen extraordinario" que consistía en implementar una estrategia de educación a distancia" (Carmona y Limón, 2020)

Por otro lado cabe señalar al respecto que en educación "Ias TIC presenciales ya se utilizan como medio para individualizar la formación de los estudiantes y buscar satisfacer las necesidades individuales. Un resultado que en las circunstancias que se presentaron podría mejorar" (Sanz, Sáinz y Capilla, 2020).

Como lo comenta González (2009) estas variaciones que experimentan los sistemas de propiedades complejas pueden llegar a situaciones en que no sean predecibles y que muy pequeñas variaciones en las condiciones iniciales, provocan grandes cambios irregulares, no periódicos, en las propiedades, cantidades o valores del sistema, en este caso en la educación Media y Superior.

Respecto a esto las investigaciones relacionadas con el conocimiento y la aceptación de las tecnologías de la información y la comunicación (TIC) en el proceso educativo, específicamente cuando los profesores las incluyen en su labor docente, van en aumento debido a su acelerada incorporación en la enseñanza; sin embargo, Sánchez y Martínez (2019) señalan que la simple introducción o presencia de las TIC en las instituciones de educación no es suficiente para una mejora en los procesos y resultados del aprendizaje.

En primer lugar tenemos al liderazgo educativo como determinante de nuestras variable independiente, se define como "[...] la labor de movilizar e influenciar a otros para articular y lograr las intenciones y 
metas compartidas de la escuela" (Leithwood, 2009, p.20) y se reconoce como la práctica del mejoramiento (Elmore, en Mineduc, 2015). Desde esta perspectiva de "práctica", el liderazgo no es un atributo o característica personal del líder, sino de un conjunto de acciones que, además, se construyen de forma situada. En esta línea, el marco para la buena dirección y liderazgo escolar (MBDLE, 2015), plantea que un liderazgo efectivo presenta variaciones según el contexto y tipo de establecimiento, como también conforme a la etapa de mejoramiento o nivel de desarrollo del establecimiento educacional.

Así mismo, el liderazgo efectivo se caracteriza por ser situacional y contingente, el MBDLE señala que liderazgo escolar se constituye en un conocimiento profesional relevante en el ejercicio de la dirección y liderazgo, y respecto de ello declara que "El conocer las concepciones contemporáneas de liderazgo escolar, permite tener una comprensión no sólo de las prácticas, tipos y características de los líderes escolares sino también de los valores y estrategias necesarios para implementar procesos de mejora escolar diferenciando contexto, nivel de desarrollo de la escuela y contingencia" (Mineduc, 2015, p. 34).

Desde una perspectiva amplia y funcional, tenemos que Leithwood (2009) plantea algunas nociones básicas acerca del liderazgo escolar, que son:

- El liderazgo existe dentro de relaciones sociales y sirve a fines sociales: Si bien los líderes son individuos, el liderazgo se enmarca en relaciones y organizaciones sociales que buscan lograr un fin a nivel colectivo. Es decir, el liderazgo no se personaliza, no es un fenómeno individual.

- El liderazgo implica un propósito y una dirección: Los líderes persiguen metas con claridad y responden por su cumplimiento.

- El liderazgo es un proceso de influencia: Se evidencia tanto en las acciones de los líderes que tienen un efecto directo en las metas del grupo, como en su capacidad para influenciar en los pensamientos y actuar de otras personas, estableciendo condiciones para ser efectivos.

- El liderazgo es una función: El liderazgo conlleva un conjunto de funciones que no necesariamente están vinculadas a una designación formal. Son diversas las personas que pueden ejercer funciones de liderazgo.

- El liderazgo es contextual y contingente, el liderazgo se practica de acuerdo a las particularidades de la organización social, las metas establecidas, las personas involucradas, los recursos y, aún más, las características del propio líder, entre otros factores. Por lo tanto, "(...) ninguna fórmula del liderazgo efectivo es aplicable de manera universal." (Leithwood, 2009, p.19)
Los términos "innovación" e "innovación educativa" aparecen cada vez con más frecuencia en el discurso sobre educación superior, de tal manera que forman ya parte integral de su estructura. Se acepta e incorpora en nuestro bagaje de premisas y supuestos, como si su significado fuera el mismo para todas las personas, grupos y ámbitos del conocimiento relacionados con el tema, ya que el uso que le damos a las palabras en conferencias, presentaciones, publicaciones y pláticas informales, genera la percepción engañosa como panorama general de la innovación educativa de que el término es globalmente aceptado y que una innovación educativa es lo que el "experto" dice, solamente porque él/ella lo dice. Sin embargo, es necesario reflexionar sobre los significados de los términos, ubicándolos en el contexto educativo (Blanco y Messina, 2000).

La tendencia reciente a incorporar la innovación educativa en las instituciones de educación superior de México y América Latina como una categoría, como una estrategia o como un propósito institucional, debe motivarnos a no aceptar acríticamente propuestas elaboradas en otros contextos y culturas, sin antes examinarlas e intentar generar nuestras propias versiones (Blanco y Messina, 2000). Sin embargo, etimológicamente, la palabra innovación proviene del latín innovatio que significa "crear algo nuevo". También se usa en el sentido de nuevas propuestas e inventos. Diversos estudios han documentado que la palabra innovación tiene significados diferentes para personas diferentes, por lo que es relevante intentar algunas aproximaciones para hablar el mismo lenguaje y poder comunicarnos sobre el tema (Magda y Buban, 2018).

La definición ligada al desarrollo de habilidades de liderazgo transformacional es la propuesta por Banerjee (2017): "Innovación es la habilidad de superar enfoques normativos con un margen significativo, producir nuevos valores, resultados, paradigmas y transformaciones". Las conductas innovadoras requieren creatividad y receptividad al cambio, aunque la creatividad por sí misma no garantiza la innovación (por ejemplo, un criminal puede ser creativo).

Se puede definir innovación como el proceso de ingresar algo nuevo dentro de una realidad preexistente, para cambiar, transformar o mejorar dicha realidad. Independientemente del concepto específico que usemos de innovación, aparentemente se trata de un proceso de múltiples etapas con el que las organizaciones transforman sus ideas en productos, procesos o servicios, para mejorar la calidad de todo el sistema (Gheh, 2009).

Finalmente la innovación educativa trasladada a la educación superior, es necesario tomar en cuenta la amplitud y profundidad del campo de estudios de la educación (Tierney y Lanford, 2016). En palabras de Shulman (1981) la educación es un campo de estudio, 
un lugar que contiene fenómenos, eventos, instituciones, problemas, personas y procesos, que en sí mismos constituyen la materia prima para hacer indagaciones de muchos tipos. Las perspectivas y procedimientos de muchas disciplinas pueden utilizarse para resolver preguntas que surgen de y que son inherentes a la educación como un campo de estudio. Mientras cada una de estas perspectivas disciplinarias se utiliza en el campo de la educación, trae consigo su propio conjunto de conceptos, métodos y procedimientos, a menudo modificándose para adaptarse a los fenómenos y problemas de la educación.

Diversos autores han definido a la innovación educativa desde distintos enfoques. Hay quienes definen innovación con un sentido instrumental, como la introducción de herramientas: "El acto de crear y difundir nuevas herramientas educativas, prácticas instruccionales, organizacionales y tecnológicas" o como la búsqueda de soluciones: "Es la acción permanente realizada mediante la investigación para buscar nuevas soluciones a los problemas planteados en el ámbito educativo", por lo tanto una innovación educativa implica la implementación de un cambio significativo en el proceso de enseñanza-aprendizaje, de los materiales empleados para el mismo, de los métodos de entrega de las sesiones, de los contenidos o en los contextos que implican la enseñanza (López y Heredia, 2017).

El concepto de innovación implica el cambio, pero mediado por tres condiciones:

1. El cambio debe ser consciente y deseado, por lo que se constituye en el resultado de una voluntad decidida y deliberada.

2. El cambio es producto de un proceso, con fases establecidas y tiempos variables.

3. El cambio no modifica sustancialmente la práctica profesional, esto es, el cambio se da dentro de los límites admisibles por la legislación y el status quo establecido. (Barraza. 2005)

Frente a la situación que se vive actualmente, lo que corresponde es encontrar los efectos y soluciones, tal como hacen referencia las posiciones de la teoría de la complejidad para lo que Guía, J. et al. (2008) dice: "Las teorías de la complejidad en el marco de la causalidad transformativa pueden servir, como base para pasar de un paradigma basado en la dualidad sistema-entorno a un nuevo paradigma fundamentado en los conceptos de identidad-diferencia. En este nuevo paradigma una innovación aparece debido a la necesidad intrínseca que los individuos tienen, individual y colectivamente, de expresar sus identidades y sus diferencias. La identidad y la diferencia emergen, convirtiéndose en lo que son a través de la causa transformativa de la auto organización, es decir, de las relaciones. La innovación emerge, por tanto, de las relaciones de sus miembros en lugar de ser determinadas por la libertad de elección de los individuos". Tal afirmación puede referirse a una forma distinta de ver la solución que involucre la participación de todo un equipo.

Por su parte, Lino (2006) comenta que "se busca finalmente superar críticamente la independencia, hegemonía, y supremacía de la ciencia con respecto a otras formas de obtención de conocimientos; el establecimiento de las categorías sujeto y objeto del conocimiento como entidades separadas y autónomas; la concepción de la investigación como descubrimiento por el sujeto de las propiedades del mundo, -ocultas como esencias-, pero existentes al margen del sujeto, objetivamente; y la concepción del método, como garante de la confiabilidad de los resultados cognoscitivos. Todo esto supone un cambio en el mundo de los conceptos, y cuestionar esos conceptos maestros con los cuales se piensa y se presiona el mundo". Esto resalta una vez más la posibilidad de encontrar estabilidad a través del pensamiento crítico y su actuación.

Por otro lado, y antes de continuar con los conceptos de nuestra variable dependiente (personal, servicio, gestión escolar y sistemas) resulta clave conceptualizar qué es un proyecto. Para ello, se tomará de referencia aquella definición establecida por la autoridad internacional del PMI (Project Management Institute) en su constructo PMBOK (Project Management Body of Knowledge), $5^{a}$ edición. Según él, "un proyecto es un esfuerzo temporal que se lleva a cabo para crear un producto, servicio o resultado único".

Aquí, se debe realizar énfasis en su carácter único (corresponde a un conjunto específico de operaciones diseñadas para el logro de una meta específica) y temporal (tiene un comienzo y un fin definido, y por ende, presenta límites y recursos bien definidos). Los orígenes de la gestión de proyectos han presentado varios hitos a lo largo de la historia.

La definición oficial proporcionada por la Asociación para la Gestión de Proyectos (APM, 2013) dice:

La gestión de proyecto se enfoca en controlar la introducción del cambio deseado.

Esto implica:

- Comprender las necesidades de los grupos de interés

- Planificar qué se necesita hacer, cuándo, por quién y bajo qué estándares

Crear y motivar al equipo

- Coordinar el trabajo de diferentes personas

- Monitorear el trabajo que se realiza

- Gestionar cualquier cambio del plan

- Alcanzar resultados satisfactorios.

Lerner y Eisik (2017), clasifican las barreras que 
quebrantan potencialmente el éxito de un proyecto hoy día:

"1. Barreras directamente controlables: aquellas sobre las cuales la gestión del proyecto puede ejercer influencia directa (por ejemplo, la comunicación).

2. Barreras sobre las cuales no existe control directo, pero se puede ejercer influencia: aquellas sobre las cuales la gestión del proyecto puede accionar, pero no controlar en su totalidad (por ejemplo, cuestiones relacionadas a la definición del alcance del proyecto).

3. Barreras sobre las que no existe control directo y no se puede ejercer influencia: por ejemplo, factores políticos (¿quién ganará las próximas elecciones?), económicos (tasa de inflación, crecimiento del PBI), sociales (niveles de pobreza, tasa de natalidad y desnutrición), tecnológicos (nivel de adopción de tecnologías de robotización de procesos, velocidad de desarrollo de nuevos desarrollos) etc)."

El Gerente de Proyectos (Project Manager o PM por sus siglas en inglés) desempeña un rol fundamental en todas las etapas del ciclo de vida de un proyecto. Según el PMI, corresponde a la persona asignada por la organización dedicada a liderar el equipo responsable de lograr los objetivos de un proyecto y cumple un rol técnico, operativo y estratégico requerido para el avance del mismo. Las principales funciones del PM se enumeran a continuación:

- Concentrar y consolidar todo el esfuerzo realizado por el equipo de trabajo y las diferentes unidades organizacionales involucradas.

- $\quad$ Ejercer el liderazgo del equipo del proyecto para alcanzar los objetivos del proyecto y de los grupos de interés. Actuar como nexo estratégico entre los equipos operativos y la Alta Dirección, ejerciendo un rol proactivo.

- Apalancarse en una Oficina de Gestión de Proyectos independiente y robustamente constituida y con un rol de gestión (no sólo administrativo) para contar con información efectiva a efectos de facilitar la toma de decisiones en Comités Ejecutivos/Directivos, donde diversos sponsors deben tomar decisiones.

- Facilitar a todos los colaboradores la visión de los objetivos y el éxito del proyecto, así como también, su dirección hacia la consecución de los mismos. Liderar esfuerzos y brindar soporte a los múltiples equipos de trabajo, oficiando de facilitador.

- Establecer las restricciones del proyecto (tiempo, presupuesto y objetivos de los interesados, tenien- do en consideración los recursos disponibles) y ejecutar los mecanismos necesarios para cumplimentarlas.

Predecir las posibles amenazas y oportunidades que puedan surgir, por intermedio de una efectiva gestión de riesgos del proyecto.

- Proveer liderazgo, planificación y coordinación a su equipo de trabajo, mediante una comunicación efectiva, tanto a nivel verbal como no verbal (planes, documentos, cronogramas, etc. ).

- Bregar por la constitución de un equipo de aseguramiento de la calidad (QA o Quality Assurance por sus siglas en inglés) que complemente la labor de la PMO.

- Facilitar y apoyar procesos de gestión del cambio durante el desarrollo del proyecto, integrando este fenómeno a lo largo de todo el proyecto."

Adentrándonos un poco más en lo que es nuestra variable dependiente (gestión de proyectos) en sus dimensiones el personal, servicio, gestión escolar y sistemas, tenemos que la gestión escolar en los establecimientos educativos, es un proceso sistemático que está orientado al fortalecimiento de las instituciones educativas y a sus proyectos, con el fin de enriquecer los procesos pedagógicos, directivos, comunitarios y administrativos; que esto va acorde de nuestras demás dimensiones de nuestra variable.

Así mismo la gestión escolar está constituida por cuatro áreas de gestión: área de gestión directiva, área de gestión pedagógica y académica, área de gestión de la comunidad y área de gestión administrativa y financiera. Por esto mismo es importante mencionar la importancia que tiene la gestión escolar para el mejoramiento de la calidad en un país, radicando en el fortalecimiento de las secretarías de educación y de los establecimientos educativos; en sus instancias administrativas y sistema pedagógico, para generar cambios y aportar un valor agregado en conocimientos y desarrollo de competencias a los estudiantes.

Sin embargo la pandemia de hoy día fue un desafío a enfrentar para todo el ámbito educativo, por ejemplo los cierres de escuelas provocados por el coronavirus afectaron desproporcionadamente a los niños y las niñas, porque no todos tuvieron las oportunidades, las herramientas o el acceso necesarios para seguir aprendiendo durante la pandemia. Para millones de estudiantes, el cierre de escuelas no será una interrupción temporal de su educación, sino un final abrupto de la misma. Por ende la educación debería estar en el centro de los planes de recuperación de todos los gobiernos, para que la educación sea gratuita y accesible a todos los niños y las niñas del mundo.

Por otro lado tenemos que, la Comisión Económica para América Latina y el Caribe (CEPAL) ha planteado 
que, incluso antes de enfrentar la pandemia, la situación social en la región se estaba deteriorando, debido al aumento de los índices de pobreza y de pobreza extrema, la persistencia de las desigualdades y un creciente descontento social.

En este contexto, la crisis tendrá importantes efectos negativos en los distintos sectores sociales, incluidos particularmente la salud y la educación, así como en el empleo y la evolución de la pobreza. Por su parte, la UNESCO ha identificado grandes brechas en los resultados educativos, que se relacionan con una desigual distribución de los docentes, en general y de los mejor calificados, en particular, en países y regiones con menores ingresos y de zonas rurales, las que suelen concentrar además a población indígena y migrante.

En el ámbito educativo, gran parte de las medidas que los países de la región han adoptado ante la crisis se relacionan con la suspensión de las clases presenciales en todos los niveles, lo que ha dado origen a tres campos de acción principales: el despliegue de modalidades de aprendizaje a distancia, mediante la utilización de una diversidad de formatos y plataformas (con o sin uso de tecnología); el apoyo y la movilización del personal y las comunidades educativas, y la atención a la salud y el bienestar integral de las y los estudiantes.

Para el estudio de lo anterior también es necesario tener en cuenta los efectos de tomar como referencia la teoría de la complejidad que ayude a descifrar los problemas y encontrar alternativas de cambio, como lo comenta Gallegos (2016) que el ámbito académico ha sido testigo de un notable crecimiento de la literatura relacionada con la temática de la complejidad. La noción de complejidad se ha transformado en una categoría teóricamente dominante. Por lo que en esta situación, abordar este concepto no puede pasar desapercibido, pues es precisamente la complejidad que la gestión estratégica de las instituciones en estos tiempos presenta, aunada a la especialización que requieren las actividades y la dificultad del medio ambiente, por lo que resulta interesante y necesario encontrar los factores involucrado en medio de la complejidad para poder dar una respuesta pronta y sólida.

\section{Método}

La metodología a emplear para esta investigación fue de tipo cuantitativa, con un diseño transaccional y un alcance correlacional, la variable independiente son los desafíos en sus dimensiones (entorno innovador, retos inesperados, liderazgo) y la variable dependiente gestión de proyectos con sus dimensiones (producto, proceso, personal, sistemas).

El diseño de nuestra investigación fue aleatorio, se sacó nuestra muestra para aplicar encuestas con nivel de confianza y margen de error, la población total de Felipe Carrillo Puerto es de 1491 docentes.

Tabla 1. Sistema educativo de Quintana Roo

\begin{tabular}{|c|c|c|}
\hline Municipio & Alumnos & Docentes \\
\hline Bacalar & 13,086 & 648 \\
\hline Benito Juárez & 196,081 & 11,233 \\
\hline Felipe Carrillo Puerto & 30,303 & 1491 \\
\hline
\end{tabular}

Fuente: Secretaría de Educación Pública (2017)

La muestra resultante fue de 66 encuestas a aplicar, con una heterogeneidad del $50 \%$, un margen de error del $10 \%$ y un $90 \%$ de nivel de confianza en el Municipio Felipe Carrillo Puerto, Quintana Roo. Se realizó una prueba piloto al instrumento y posteriormente se aplicaron las 66 globales para la investigación, para la base de datos y el vaciado de las respuestas se utilizó el programa SPSS. Se diseñó un análisis estadístico correlacional, se utilizó la herramienta SPSS para dichos análisis, se sacaron gráficas y a partir de ellas los porcentajes correspondientes a las preguntas clave de nuestro cuestionario. Teniendo así respuesta al objetivo planteado de esta investigación.

\section{Resultados}

A continuación se muestra el perfil de los encuestados en donde se observa que el $87.88 \%$ está representado por docentes como a continuación se observa en la tabla 2.

Tabla 2. Puesto de los encuestados

\begin{tabular}{|c|c|}
\hline Puesto & Porcentajes \\
\hline Docente & $87.88 \%$ \\
\hline Administrativo & $4.55 \%$ \\
\hline Jefatura & $3.03 \%$ \\
\hline Subdirectora & $3.03 \%$ \\
\hline Directora & $1.51 \%$ \\
\hline
\end{tabular}

Fuente: elaboración propia

En lo que respecta al género, en los encuestados el $50 \%$ fueron de sexo femenino como masculino, como se puede observar en la tabla 3 .

Tabla 3. Género

\begin{tabular}{|c|c|}
\hline Género & Porcentaje \\
\hline Femenino & $50 \%$ \\
\hline Masculino & $50 \%$ \\
\hline
\end{tabular}

Fuente: elaboración propia 
En lo que respecta a la edad de los encuestados el $34.84 \%$ su edad está entre los 36 a 45 años de edad como se puede observar en la tabla 4.

Tabla 4. Edad

\begin{tabular}{|c|c|}
\hline Rango de edad & Porcentaje \\
\hline 25 a 35 & $30.30 \%$ \\
\hline 36 a 45 & $34.84 \%$ \\
\hline 46 a 55 & $30.34 \%$ \\
\hline 56 a 65 & $4.54 \%$ \\
\hline
\end{tabular}

Fuente: elaboración propia

El $46.97 \%$ de los docentes encuestados tienen un grado de estudios de licenciatura y el $45.45 \%$ tienen grado de maestría y solo el $7.58 \%$ cuentan con un doctorado como se puede observar en la tabla 5.

Tabla 5. Escolaridad

\begin{tabular}{|c|c|}
\hline Grado de escolaridad & Porcentaje \\
\hline Licenciatura & $46.97 \%$ \\
\hline Maestría & $45.45 \%$ \\
\hline Doctorado & $7.58 \%$ \\
\hline \multicolumn{2}{|r|}{ Fuente: elaboración propia } \\
\hline
\end{tabular}

En cuanto a la antigüedad de los docentes encuestados el $60.61 \%$ tiene por lo menos de 1 a 10 años laborando dentro de la institución, el $21.21 \%$ cuenta con al menos de 11 a 20 años como se muestra en la tabla 6.

Tabla 6. Tiempo laborando en su institución

\begin{tabular}{|c|c|}
\hline Antigüedad & Porcentaje \\
\hline 1 a 10 & $60.61 \%$ \\
\hline 11 a 20 & $21.21 \%$ \\
\hline 21 a 30 & $18.18 \%$ \\
\hline
\end{tabular}

Fuente: elaboración propia

En los niveles educativos en los que se encuentran impartiendo clases los docentes son en un $50 \%$ a nivel universidad y el $48.48 \%$ en preparatoria y solo el $1.52 \%$ imparte en ambos niveles como se muestra en la tabla 7.

Tabla 7. Nivel al que imparte clases

\begin{tabular}{|c|c|}
\hline Niveles educativos & Porcentaje \\
\hline Preparatoria & $48.48 \%$ \\
\hline Universidad & $50 \%$ \\
\hline Ambos & $1.52 \%$ \\
\hline
\end{tabular}

Fuente: elaboración propia
En cuanto a los principales desafíos que enfrentan los docentes de Felipe Carrillo Puerto de Quinta Roo encontramos que en primer lugar, se encuentran los retos inesperados (forma de impartir clases, manera de relacionarse con los alumnos) con un $75 \%$ en las respuestas de casi siempre y siempre. En segundo lugar, se encuentra la dimensión del liderazgo (motivación a los alumnos) con un $69 \%$ en las respuestas de casi siempre y siempre y en tercer lugar, la dimensión del entorno innovador (cambios en el plan de trabajo) con un 39\% en las respuestas de casi siempre y siempre como se muestra en la tabla 8.

Tabla 8. Desafíos que enfrentan los docentes en Felipe Carrillo Puerto

\begin{tabular}{|c|c|}
\hline Variable & Porcentaje \\
\hline Retos inesperados & $75 \%$ \\
\hline Liderazgo & $69 \%$ \\
\hline Entorno innovador & $39 \%$ \\
\hline
\end{tabular}

Fuente: elaboración propia

En lo que respecta a la gestión escolar respecto a la administración escolar, los docentes de las escuelas de Felipe Carrillo Puerto, Quintana Roo, en sus dimensiones el orden que asignaron en cuanto al nivel de importancia fue el siguiente: En primer lugar, con un empate se encuentran el personal y sistemas con un $90 \%$ ambos en la respuesta de muy importante e importante, En segundo lugar, la dimensión de gestión escolar, con un $88 \%$ en la respuesta de muy importante e importante y finalmente en tercer lugar, se encuentra la dimensión servicio, con un $87 \%$ en la respuesta de muy importante e importante.

Tabla 9. Importancia de las variables de gestión escolar en la administración escolar

\begin{tabular}{|c|c|}
\hline Variable & Porcentaje \\
\hline Personal & $90 \%$ \\
\hline Sistemas & $90 \%$ \\
\hline Gestión escolar & $88 \%$ \\
\hline Servicio & $87 \%$ \\
\hline
\end{tabular}

Fuente: elaboración propia

Al hacer un cruce de variables entre la importancia que tiene el entorno innovador (cambios en el plan de impartir clases, asesoramiento, etc.) en las dimensiones de la variable gestión de proyectos (administración escolar) como se observa en la tabla 10: 
En primer lugar, el personal en un $90 \%$ con totalmente y mucho.

En segundo lugar, los sistemas $85 \%$ con totalmente y mucho.

En tercer lugar, la gestión escolar, 79\% con totalmente y mucho.

En cuarto lugar, el servicio en un $78 \%$ con totalmente y mucho.

Tabla 10. Importancia del entorno innovador en las dimensiones de la variable de gestión de proyectos

\begin{tabular}{|c|c|}
\hline Variable & Porcentaje \\
\hline Personal & $90 \%$ \\
\hline Sistemas & $85 \%$ \\
\hline Gestión escolar & $79 \%$ \\
\hline Servicio & $78 \%$ \\
\hline
\end{tabular}

Fuente: elaboración propia

Al hacer un cruce de variables entre la importancia que tienen los retos inesperados (forma presencial a virtual, etc.) en las dimensiones de la variable gestión de proyectos se encontró en primer lugar, el personal, $91 \%$ con totalmente y mucho, en segundo lugar, los sistemas $83 \%$ con totalmente y mucho, en tercer lugar, el servicio $81 \%$ con totalmente y mucho y finalmente en cuarto lugar, la gestión escolar $79 \%$ con totalmente y mucho como se observa en la tabla 11.

Tabla 11. Importancia de los retos inesperados en las dimensiones de la variable de gestión de proyectos

\begin{tabular}{|c|c|}
\hline Variable & Porcentaje \\
\hline Personal & $91 \%$ \\
\hline Sistemas & $83 \%$ \\
\hline Servicio & $81 \%$ \\
\hline Gestión escolar & $79 \%$ \\
\hline
\end{tabular}

Fuente: elaboración propia

Al hacer un cruce de variables entre la importancia que tienen los retos de liderazgo (motivación) en las dimensiones de la variable gestión de proyectos se encontró en primer lugar, el personal $88 \%$ con totalmente y mucho, en segundo lugar, los sistemas $82 \%$ con totalmente y mucho, en tercer lugar, el servicio $78 \%$ con totalmente y mucho y en cuarto lugar, gestión escolar $73 \%$ con totalmente y mucho.
Tabla 12. Importancia de los retos de liderazgo en las dimensiones de las variables de gestión de proyectos

\begin{tabular}{|c|c|}
\hline Variable & Porcentaje \\
\hline Personal & $88 \%$ \\
\hline Sistemas & $82 \%$ \\
\hline Servicio & $78 \%$ \\
\hline Gestión escolar & $73 \%$ \\
\hline
\end{tabular}

Fuente: elaboración propia

\section{Conclusiones}

La mayoría de los autores de literatura de gestión de proyecto concuerdan en que la gestión de proyecto se trata de establecer y, después, alcanzar (o superar) objetivos de tiempo, costo y desempeño (calidad). Progresivamente, los autores incluyen la variable de seguridad, que cobra más y más importancia como resultado del aumento de disposiciones estatutarias sobre salud y seguridad. Algunos autores también incluyen la variable de riesgo, pues la necesidad económica conduce, cada vez más, a los proyectos hacia estados de riesgo más y más altos, en este caso la gestión de un proyecto es necesaria para dar respuesta a la teoría de la complejidad en donde a través de esta teoría se pudo observar con claridad los desafíos que enfrentan los docentes donde el $50 \%$ fueron de instituciones de Media Superior y el $50 \%$ de nivel Superior en Felipe Carrillo Puerto, Quintana Roo, derivados de la contingencia COVID-19 concatenado a la complejidad del contexto y que permite a su vez encontrar cómo a través de la adecuada gestión se puede manejar ese caos, mostrando que desafíos formas de administrar se puede mejorar en ellas.

Finalmente puede concluir que el principal desafío que enfrentan los docentes de esta región frente al periodo pandémico en primer lugar con un $75 \%$ se encuentran los retos inesperados los cuales que tiene que ver con la forma de impartir clases y la manera de relacionarse con los alumnos, dando claridad a la imperiosa necesidad de modificar en este sentido la forma tradicional de impartir un curso, a la utilización de tecnologías de la información y que para ello los docentes deben estar capacitados en este tema y con ello lograr una adecuada planeación de sus clases, así mismo se requiere de un establecimiento de una comunicación asertiva y bidireccional en donde los docentes puedan retroalimentar a sus estudiantes sobre su desenvolvimiento en el transcurso de los cursos impartidos.

El segundo desafío que deben enfrentar los docentes con un $69 \%$ es el liderazgo en relación a encontrar una adecuada motivación para con sus estudiantes en esa modalidad virtual tarea que para nada es sencilla ya 
que en muchos de los casos deben tener presente el cambio generacional de los mismos para saber de qué manera lograr motivar a cada estudiante y lograr hacer esa empatía con sus grupos en donde pueda mantener a sus grupos interesados en sus cursos.

El tercer desafío es desarrollar un entorno innovador en un $39 \%$ lo cual implica realizar cambios en el plan de trabajo a como se venía realizando anteriormente cuando las clases eran presenciales a una planeación donde el docente pueda desarrollar estrategias de enseñanza aprendizaje en base a las competencias pero considerando tanto el ambiente externo como el interno, estrategias de evaluación, evidencias e desempeño y rubricas con la intención de dejar claridad en las actividades que se planean.

la variable independiente son los desafíos en sus dimensiones (entorno innovador, retos inesperados, liderazgo) y la variable dependiente gestión de proyectos con sus dimensiones (producto, proceso, personal, sistemas).

En lo que respecta a la variable dependiente que fue la gestión de proyectos, en sus dimensiones (producto, proceso, personal, sistemas) para poder enfrentar los retos, principalmente deben trabajar con el personal y sus sistemas con un $90 \%$ en ambos, ya que los docentes de Felipe Carrillo Puerto colocan en totalmente importante al personal, por lo que será necesario priorizar en medio de la contingencia y en momentos futuros por la adversidades que se pudieran presentar y que se traducen como las dificultades al accionar tal como lo indica la teoría de la complejidad, para lo que es en estas condiciones algo inevitable y que avasalla ante la presencia de lo imprevisto, por lo cual se recomienda prestar atención al factor humano principalmente para salir avante en todo momento y cada IEMS y Superior realizar sus propias estrategias para lograr impactar a corto, mediano y largo plazo en sus instituciones para lograr mantener la calidad en todo momento a pesar de los retos.

\section{Referencias}

Arias-Pineda, A.A. y Ramirez-Martinez, L. (2019). La organización-empresa: ¿un sistema vivo? Aportes de la teoría de la complejidad y la filosofía ambiental a la teoría administrativa y organizacional. Revista Escuela de Administración de Negocios, (86), 133-150. DOI: https://doi.org/10.21158/01208160.n86.2019.2298.

Barberousse, P. (2008). Fundamentos Teóricos Del Pensamiento Complejo De Edgar Morin. Revista Electrónica Educare, XII(2), 95-113. Disponible en: https://www. redalyc.org/articulo.oa?id=194114586009
Barraza Macías, A. Una conceptualización comprehensiva de la innovación educativa. Innovación Educativa, 5(28), 19-31. Disponible en: https://www.redalyc.org/ pdf/1794/179421470003.pdf.

Blanco, R., y Messina, G. (2000). Estado del arte sobre las innovaciones educativas en América Latina. Colombia: Convenio Andrés Bello, UNESCO.

Cárdenas, M. y Rivera, J. (2004) La teoría de la complejidad y su influencia en la escuela. Revista de Teoría y Didáctica de las Ciencias Sociales, (9),131-141. Disponible en:https://www.redalyc.org/pdf/652/65200908. pdf

Carmona I y Limón C. (ABRIL 22, 2020). "Los maestros frente al Covid-19". Disponible en:https://educacion. nexos.com.mx/los-maestros-frente-al-covid-19/

Cervantes, E (2020, 02 mayo) Resistir la Covid-19. Intersecciones en la Educación de Ciudad Juárez, México. Disponible en: http://cathi.uacj.mx/handle/20.500.11961/11665

Comisión Económica para América Latina y el Caribe (CEPAL, agosto 2018). La educación en tiempos de la pandemia de COVID-19. Disponible en: : https://www. cepal.org/es/publicaciones/45904-la-educacion-tiempos-la-pandemia-covid-19

Darchibald, R. (1989). Project management education and training in the USA. International Journal of Project Management. 7 (4). 199-200. DOI: https://doi. org/10.1016/0263-7863(89)90003-3

División Educación General. LIDERAZGO ESCOLAR: Reconociendo los tipos de liderazgo. Disponible en:https:// liderazgoescolar.mineduc.cl/wp-content/uploads/sites/55/2019/05/HERRAMIENTA2_final.pdf

Ferrás, X. (2010). Innovación 6.0: el fin de la estrategia. Barcelona: Plataforma Editorial.

Gallegos, M. (2016) Una cartografía de las ideas de la complejidad en América Latina: la difusión de Edgar Morin. Revista de Estudios Latinoamericanos, 63, 93128. Disponible en: https://www.sciencedirect.com/ science/article/pii/S1665857416300266

González, J. (2009). La teoría de la complejidad. Dyna, 76(157), 243-245. Disponible en: https://www.redalyc.org/articulo.oa?id=49611942024

Guia, J., Prats, L. y Comas, J. (2009). Innovación como cambio institucional: una aproximación desde las teorías de la complejidad. Investigaciones Europeas de Dirección y Economía de la Empresa, 15, (2), 93104. Disponible en:https://www.sciencedirect.com/ science/article/pii/S1135252312600914Leithwood, K. (2009) ¿Cómo liderar nuestras escuelas? Aportes desde la Investigación. Santiago

Universidad de Chile: Fundación Chile.

Lerner A. y Eisik M. (2017). Gestión de proyectos: desafíos y recomendaciones prácticas para su aplicación en las organizaciones. Checkpoint. 1-9. [Fecha de Consulta 26 de Julio de 2021]. Disponible en: https://www.eco- 
nomicas.unsa.edu.ar/afinan/afe_1/material_de_estudio/material/Gestion\%20de $\% 20$ proyectos $\% 20$ desafios $\% 20 y \% 20$ recomendaciones $\% 20$ practicas $\% 20$ para\%20su\%20aplicacion\%20en\%20las\%20organizaciones.pdf

Lino, M. (2006). De la teoría de la complejidad a la filosofía intercultural: hacia un nuevo saber. Revista de Filosofía 24 (52), 65-82. Disponible en: http:// ve.scielo.org/scielo.php?script=sci_arttext\&pi $\mathrm{d}=$ S0798-11712006000100004

Magda, A. J., y Buban, J. (2018). The state of innovation in higher education: A survey of academic administrators. Louisville, KY: The Learning House, Inc. Recuperado de: https://onlinelearningconsortium.org/read/ state-of-innovation-in-higher-education/

Mejia, O. (junio, 2020). El retorno al trabajo después del COVID-19. [Forbes]. Recuperado 19 de agosto de 2020, de https://www.forbes.com.mx/el-retorno-al-trabajo-despues-del-covid-19

Narváez, C. (marzo, 2021). Los retos como responsable del Programa Emprendedor en el Centro Universitario UAEM Valle de Teotihuacán frente al Covid-19. Recuperado 24 de junio de 2021 de http://web.uaemex. mx/identidad/docs/cronicas/2021/TOMO_XIX/19.\%20 Los\%20retos\%20como\%20responsable\%20del\%20 Programa\%20Emprendedor\%20en\%20el\%20CU\%20 Teotihuacan.pdf

Parra, M. (2014). EMPRENDER en tiempos de crisis. Recuperado 24 de junio de 2021 de https://www.observatorioeconomico.cl/index.php/oe/article/view/130/133

Samperio-Pacheco, V. M. y Barragán-López, J. F. (2018). Análisis de la percepción de docentes, usuarios de una plataforma educativa a través de los modelos tpack, samr y tam3 en una institución de educación superior. Revista de innovación educativa, 10(1): 116-131. http://www.udgvirtual.udg.mx/apertura/index.php/ apertura/article/view/1162/859.

Sánchez M. y Escamilla J. (2018). Perspectivas de la innovación educativa en universidades de México: Experiencias y reflexiones de la RIE 360. Recuperado de: https://cuaieed.unam.mx/descargas/investigacion/
Perspectivas-de-la-innovacion-educativa-en-universidades-de-Mexico.pdf

Sánchez, M. y Martínez, A. (Eds.). (2019). Formación Docente en la UNAM: Antecedentes y la Voz de su Profesorado. Coordinación de Desarrollo Educativo e Innovación Curricular, UNAM.

Sancho, J y Cano, C (comps.) (2012). La fugacidad de las políticas, la inercia de las prácticas: la educación y las tecnologías de la información y la comunicación. Barcelona: Ediciones Octaedro, SL.

Sanz, I., Sáinz González, J., y Capilla, A. (2020). Efectos de la Crisis del Coronavirus en la Educación Superior. Organización de Estados Iberoamericanos para la Educación, la Ciencia y la Cultura (oei). https://oei.org.br/ arquivos/informe-covid-19d.pdf.

Servín A y Ortega P. (30 de junio de 2020). Los retos que enfrenta la educación ante la pandemia. Recuperado de: https://www.eleconomista.com.mx/gestion/ Los-retos-que-enfrenta-la-educacion-ante-la-pandemia-20200630-0039.html

Terribili, A., Bortoleto, A. C., y Bentancor, A. L. (2015). Gestión de proyectos de innovación en las instituciones educativas privadas en San Pablo. REXE. Revista de Estudios y Experiencias en Educación, 14(27), 85103. Disponible en: https://www.redalyc.org/articulo. oa? id=243143345006

Tintoré, M. (2018). Líderes educativos y justicia social. Un estudio comparativo. Perspectiva educacional. 57 (2). Disponible en: http://www.perspectivaeducacional.cl/ public/journals/1/articulos_prensa/n57_2/736.pdf

Valenzuela, P. y Horn, A. (2012). Influencia del liderazgo directivo en los resultados de los estudiantes. En J. Weinstein y G. Muñoz (Eds.), ¿Qué sabemos de los directores de Escuela en Chile? (pp.325- 348). CEPPE, Centro de Innovación en Educación Fundación Chile y Pontificia Universidad Católica de Chile. Recuperado de http://ww2.educarchile.cl/UserFiles/P0001/File/CR_ Articulos/Directores_de_Chile_INTRODUCCION.pdf

Wallace, W. (2014). Gestión de proyectos. Edinburgh, Business School. 17-19. [Fecha de Consulta 26 de Julio de 2021]. Disponible en: https://ebs.online.hw.ac.uk/ documents/course-tasters/spanish/pdf/pr-bk-taster.pdf 